\title{
Characterizations of the solution sets of pseudoinvex programs and variational inequalities
}

\author{
Caiping Liu ${ }^{1}$, Xinmin Yang ${ }^{2^{*}}$ and Heungwing Lee ${ }^{3}$
}

\author{
* Correspondence: xmyang@cqnu. \\ edu.cn \\ 2Department of Mathematics, \\ Chongqing Normal University, \\ Chongqing 400047, China \\ Full list of author information is \\ available at the end of the article
}

\begin{abstract}
A new concept of nondifferentiable pseudoinvex functions is introduced. Based on the basic properties of this class of pseudoinvex functions, several new and simple characterizations of the solution sets for nondifferentiable pseudoinvex programs are given. Our results are extension and improvement of some results obtained by Mangasarian (Oper. Res. Lett., 7, 21-26, 1988), Jeyakumar and Yang (J. Optim. Theory Appl., 87, 747-755, 1995), Ansari et al. (Riv. Mat. Sci. Econ. Soc., 22, 31-39, 1999), Yang (J. Optim. Theory Appl., 140, 537-542, 2009). The concepts of Stampacchia-type variational-like inequalities and Minty-type variational-like inequalities, defined by upper Dini directional derivative, are introduced. The relationships between the variational-like inequalities and the nondifferentiable pseudoinvex optimization problems are established. And, the characterizations of the solution sets for the Stampacchia-type variational-like inequalities and Minty-type variational-like inequalities are derived.
\end{abstract}

Keywords: Solution sets, Pseudoinvex programs, Variational-like inequalities, Optimization problem, Dini directional derivatives

\section{Introduction}

Characterizations and properties of the solution sets are useful for understanding the behavior of solution methods for programs that have multiple optimal solutions. Mangasarian [1] presented several characterizations of the solution sets for differentiable convex programs and applied them to study monotone linear complementarity problems. Since then, various extensions of these solutions set characterizations to nondifferentiable convex programs, infinite-dimensional convex programs, and multiobjective convex programs have been given in [2-4]. Moreover, Jeyakumar and Yang [5] extended the results in [1] to differentiable pseudolinear programs; Ansari et al. [6] extended the results in [5] to differentiable $\eta$-pseudolinear programs; Yang [7] extended the results in $[1,5,6]$ to differentiable pseudoinvex programs. In this paper, we extend the results in $[1,5-7]$ to the nondifferentiable pseudoinvex programs and give the characterizations of solution sets for nondifferentiable pseudoinvex programs.

The variational inequalities are closely related to optimization problems. Under certain conditions, a variational inequality and an optimization problem are equivalent. As Mancino and Stampacchia [8] pointed out: if $\Gamma$ is an open and convex subset of $R^{n}$

\section{SpringerOpen ${ }^{\circ}$}

(C) 2011 Liu et al; licensee Springer. This is an Open Access article distributed under the terms of the Creative Commons Attribution License (http://creativecommons.org/licenses/by/2.0), which permits unrestricted use, distribution, and reproduction in any medium, provided the original work is properly cited. 
and $F: \Gamma \rightarrow R^{n}$ is the gradient of the differentiable convex function $f: \Gamma \rightarrow R$, then the variational inequality (VI): find $\bar{x} \in \Gamma$, such that

$$
(y-\bar{x})^{T} F(\bar{x}) \geq 0, \quad \forall y \in \Gamma,
$$

is equivalent to the optimization problem $\{\min f(x), x \in \Gamma\}$. An important generalization of variational inequalities is variational-like inequalities (VLI): find $\bar{x} \in \Gamma$, such that

$$
\eta(y, \bar{x})^{T} F(\bar{x}) \geq 0, \quad \forall y \in \Gamma,
$$

where $\eta: \Gamma \times \Gamma \rightarrow R^{n}$. Parida et al. [9] studied the existence of the solution of (VLI) and established the relationships between the variational-like inequalities (VLI) and differentiable convex programs. But the objective function of an optimization problem is not always differentiable; therefore, the variational inequalities defined by the directional derivative are introduced. For example, Crespi et al. [10,11] introduced the Minty variational inequalities defined by lower Dini derivative and established the relationships between Minty variational inequalities and optimization problems. Later, Ivanov [12] established the relationships between variational inequalities and nondifferentiable pseudoconvex optimization problems and applied them to obtain the characterizations of solution sets of Stampacchia variational inequalities and Minty variational inequalities. In this paper, we introduce Stampacchia-type variational-like inequalities and Minty-type variational-like inequalities defined by upper Dini directional derivative, and based on the relationships between variational-like inequalities and optimization problems, we give the characterizations of solution sets of these two classes of variational-like inequalities.

\section{Preliminaries}

In this paper, let $R^{n}$ be a real $n$-dimensional Euclidean space, $\Gamma$ a nonempty subset of $R^{n}, f: \Gamma \rightarrow R$ a real-valued function, and $\eta: \Gamma \times \Gamma \rightarrow R^{n}$ a vector-valued function. Firstly, we recall some notions that will be used throughout the paper.

Definition 2.1. (See [13]) $A$ set $\Gamma$ is said to be $\eta$-invex if, for any $x, y \in \Gamma$ and any $\lambda$ $\in[0,1]$

$$
y+\lambda \eta(x, y) \in \Gamma .
$$

Definition 2.2. (See [13]) A function $f: \Gamma \rightarrow R$ is said to be preinvex with respect to $\eta$ on the $\eta$-invex set $\Gamma$ if, for any $x, y \in \Gamma$ and any $\lambda \in[0,1]$,

$$
f(y+\lambda \eta(x, y)) \leq \lambda f(x)+(1-\lambda) f(y) .
$$

Definition 2.3. (See [14]) A function $f: \Gamma \rightarrow R$ is said to be prequasiinvex with respect to $\eta$ on the $\eta$-invex set $\Gamma$ if, for any $x, y \in \Gamma$ and any $\lambda \in[0,1]$,

$$
f(y+\lambda \eta(x, y)) \leq \max \{f(x), f(y)\} .
$$

Definition 2.4. (See [15]) A function $f: \Gamma \rightarrow R$ is said to be semistrictly prequasiinvex with respect to $\eta$ on the $\eta$-invex set $\Gamma$ if, for any $x, y \in \Gamma$ with $f(x) \neq f(y)$ and any $\lambda \in$ $[0,1]$,

$$
f(y+\lambda \eta(x, y))<\max \{f(x), f(y)\} .
$$


Condition A (See [16]):

$$
f(y+\eta(x, y)) \leq f(x), \quad \forall x, y \in \Gamma .
$$

Condition C (See [14]): Let $\Gamma$ be an $\eta$-invex set. For any $x, y \in \Gamma$ and any $\lambda \in[0,1]$,

$$
\begin{aligned}
& \eta(y, y+\lambda \eta(x, y))=-\lambda \eta(x, y), \\
& \eta(x, y+\lambda \eta(x, y))=(1-\lambda) \eta(x, y) .
\end{aligned}
$$

Obviously, the map $\eta(x, y)=x-y$ satisfies Conditions A and C.

Remark 2.1. (See [16]) If Condition $C$ is satisfied, then

$$
\begin{aligned}
\eta\left(y+\lambda_{1} \eta(x, y), y+\lambda_{2} \eta(x, y)\right) & =\left(\lambda_{1}-\lambda_{2}\right) \eta(x, y), \quad \forall \lambda_{1}, \lambda_{2} \in(0,1) \\
\eta(y+\lambda \eta(x, y), y) & =\lambda \eta(x, y), \quad \forall \lambda \in[0,1] .
\end{aligned}
$$

Definition 2.5. The upper Dini directional derivative of $f$ at $x \in \Gamma$ in the direction $d$ $\in R^{n}$ is defined by

$$
f_{+}^{\prime}(x ; d)=\limsup _{t \rightarrow 0^{+}} \frac{f(x+t d)-f(x)}{t},
$$

which is an element of $R \cup\{ \pm \infty\}$.

Definition 2.6. (See [17]) A function $h: R^{n} \rightarrow R$ is said to be:

(1) positively homogeneous if $\forall x \in R^{n}, \forall r>0$, one has $h(r x)=r h(x)$;

(2) subodd if $\forall x \in R^{n} \backslash\{0\}$, one has $h(x)+h(-x) \geq 0$.

It is clear that the upper Dini directional derivative is positively homogeneous with respect to the direction $d \in R^{n}$.

Definition 2.7. The function $f: \Gamma \rightarrow R$ is called radially upper semicontinuous on the $\eta$-invex set $\Gamma$, if the function $g(\lambda):=f(y+\lambda \eta(x, y))$ is upper semicontinuous on $[0,1]$, for any $x, y \in \Gamma$.

Next, we give a mean value theorem.

Theorem 2.1. (Mean Value Theorem) Let $f: \Gamma \rightarrow R$ be radially upper semicontinuous on the $\eta$-invex set $\Gamma$, and let $f$ satisfy Condition A. Then, for any $x, y \in \Gamma$, there exists a point $u \in\{y+\lambda \eta(x, y): \lambda \in[0,1)\}$, such that

$$
f(x)-f(y) \geq f_{+}^{\prime}(u ; \eta(x, y)) .
$$

Proof. Let $g:[0,1] \rightarrow R$ be a function defined by

$$
g(\lambda):=f(y+\lambda \eta(x, y))
$$

Then, for any $\lambda \in[0,1)$,

$$
\begin{aligned}
g_{+}^{\prime}(\lambda ; 1) & =\limsup _{t \rightarrow 0^{+}} \frac{g(\lambda+t)-g(\lambda)}{t} \\
& =\limsup _{t \rightarrow 0^{+}} \frac{f(y+(\lambda+t) \eta(x, y))-f(\gamma+\lambda \eta(x, y))}{t} \\
& =f^{\prime}{ }_{+}(\gamma+\lambda \eta(x, y) ; \eta(x, y)) .
\end{aligned}
$$

Let $p:[0,1] \rightarrow R$ be another function defined by

$$
p(\lambda):=g(\lambda)+\lambda(f(y)-f(y+\eta(x, y))) .
$$


It follows from radially upper semicontinuity of $f$ that $p$ is upper semicontinuous on $[0,1]$. Note that $p(0)=p(1)=f(y)$. Therefore, $p$ attains maximum at some point $\bar{\lambda} \in[0,1)$, which implies

$$
p_{+}^{\prime}(\bar{\lambda} ; 1)=\limsup _{t \rightarrow 0^{+}} \frac{p(\bar{\lambda}+t)-p(\bar{\lambda})}{t} \leq 0 .
$$

From (2.2) and (2.1), we have

$$
p_{+}^{\prime}(\bar{\lambda} ; 1)=g_{+}^{\prime}(\bar{\lambda} ; 1)+f(y)-f(\gamma+\eta(x, y))=f_{+}^{\prime}(y+\bar{\lambda} \eta(x, y) ; \eta(x, y))+f(y)-f(y+\eta(x, y)) .
$$

From (2.3) and (2.4), we have

$$
f(\gamma+\eta(x, y))-f(\gamma) \geq f_{+}^{\prime}(y+\bar{\lambda} \eta(x, y) ; \eta(x, y)) .
$$

Set $u=y+\bar{\lambda} \eta(x, y)$, then $u \in\{y+\lambda \eta(x, y): \lambda \in[0,1)\}$. By Condition A, we obtain

$$
f(x)-f(y) \geq f_{+}^{\prime}(u ; \eta(x, y)) .
$$

Remark 2.2. In Theorem 2.1, $u \in\{y+\lambda \eta(x, y): \lambda \in[0,1)\}$ means that there exists $\bar{\lambda} \in[0,1)$, such that $u=\gamma+\bar{\lambda} \eta(x, y)$.

\section{Pseudoinvexity}

In this section, we introduce the concept of pseudoinvexity defined by upper Dini directional derivative and give some properties for this class of pseudoinvexity.

Definition 3.1. (See [18]) The function $f: \Gamma \rightarrow R$ is said to be pseudoconvex on convex set $\Gamma$ if

$$
x, y \in \Gamma, f_{+}^{\prime}(y ; x-y) \geq 0 \Rightarrow f(x) \geq f(y) .
$$

The following concept of pseudoinvexity is a natural extension for pseudoconvexity.

Definition 3.2. The function $f: \Gamma \rightarrow R$ is said to be pseudoinvex with respect to $\eta$ on the $\eta$-invex set $\Gamma$ if

$$
x, y \in \Gamma, f_{+}^{\prime}(y ; \eta(x, y)) \geq 0 \Rightarrow f(x) \geq f(y) .
$$

By Definitions 3.1 and 3.2, it is clear that every pseudoconvex function is pseudoinvex function with respect to $\eta(x, y)=x-y$, but the converse is not true.

Examples 3.1 and 3.2 will show that a pseudoinvex function with respect to a given mapping $\eta: \Gamma \times \Gamma \rightarrow R^{n}$ is not necessarily pseudoconvex function.

Example 3.1. Let $\Gamma=\left\{\left(x_{1}, x_{2}\right) \in R^{2}: x_{2} \in\left(-\frac{\pi}{2}, \frac{\pi}{2}\right)\right\}$, and let $f: \Gamma \rightarrow R, \eta: \Gamma \times \Gamma \rightarrow$ $R^{2}$ be functions defined by

$$
\begin{aligned}
f\left(x_{1}, x_{2}\right) & =x_{1}+\sin x_{2}, \\
\eta(x, y) & =\left(x_{1}-y_{1}, \frac{\sin x_{2}-\sin y_{2}}{\cos y_{2}}\right),
\end{aligned}
$$

where $x=\left(x_{1}, x_{2}\right) \in \Gamma, y=\left(y_{1}, y_{2}\right) \in \Gamma$. Then, $f$ is pseudoinvex with respect to the given mapping $\eta$. But it is not pseudoconvex, because there exist $x=\left(1+\frac{\sqrt{2}}{4} \pi,-\frac{\pi}{4}\right)$, $y=\left(1, \frac{\pi}{4}\right)$, such that $f_{+}^{\prime}(y ; x-y)=0$, but $f(x)=\frac{\sqrt{2}}{4} \pi+1-\frac{\sqrt{2}}{2}<1+\frac{\sqrt{2}}{2}=f(y)$. 
Example 3.2. Let $f: R \rightarrow R, \eta: R \times R \rightarrow R$ be defined by

$$
\begin{aligned}
& f(x)=-|x|, \quad x \in R ; \\
& \eta(x, y)=\left\{\begin{array}{lll}
x-y, & x \geq 0, y \geq 0 & \text { or } \quad x \leq 0, y \leq 0 \\
y, & x>0, y<0 & \text { or } \quad x<0, y>0 .
\end{array}\right.
\end{aligned}
$$

Then, $f$ is pseudoinvex with respect to the given mapping $\eta$. But it is not pseudoconvex, because there exist $x=-5, y=4$, such that $f_{+}^{\prime}(y ; x-y)=9>0$, but $f(x)=-5<-4$ $=f(y)$.

Definition 3.3. (See [19]) The bifunction $h: \Gamma \times R^{n} \rightarrow R$ is said to be pseudomonotone on if, for any $x, y \in \Gamma$,

$$
h(x ; y-x) \geq 0 \Rightarrow h(y ; x-y) \leq 0 .
$$

We extend this pseudomonotonicity to $\eta$-pseudomonotonicity.

Definition 3.4. Let $\Gamma \times \Gamma \rightarrow R^{n}$ be a given mapping. The bifunction $h: \Gamma \times R^{n} \rightarrow R \cup$ $\{ \pm \infty\}$ is said to be $\eta$-pseudomonotone on $\Gamma$ if, for any $x, y \in \Gamma$,

$$
h(x ; \eta(y, x)) \geq 0 \Rightarrow h(y ; \eta(x, y)) \leq 0 .
$$

The above implication is equivalent to the following implication:

$$
h(y ; \eta(x, y))>0 \Rightarrow h(x ; \eta(y, x))<0 .
$$

Next, we present some properties for pseudoinvexity.

Theorem 3.1. Let $f: \Gamma \rightarrow R$ be radially upper semicontinuous on the $\eta$-invex set $\Gamma$. Suppose that

(1) $f$ is a pseudoinvex function with respect to $\eta$ on $\Gamma$;

(2) $f$ and $\eta$ satisfy Conditions $A$ and $C$, respectively;

(3) $f_{+}^{\prime}$ is subodd in the second argument.

Then, $f$ is a prequasiinvex function with respect to the same $\eta$ on $\Gamma$.

Proof. Suppose, on the contrary, $f$ is not prequasiinvex with respect to $\eta$ on $\Gamma$. Then, $\exists x, y \in \Gamma, \exists \bar{\lambda} \in(0,1]$, such that

$$
f(y+\bar{\lambda} \eta(x, y))>\max \{f(x), f(y)\} .
$$

Without loss of generality, let $f(y) \geq f(x)$, then

$$
f(y+\bar{\lambda} \eta(x, y))>f(y) \geq f(x) .
$$

From radially upper semicontinuity of $f,(3.1)$ and Condition A, there exists $\lambda^{*} \in(0$, 1) such that

$$
f\left(y+\lambda^{*} \eta(x, y)\right)=\max _{\lambda \in[0,1]}\{f(y+\lambda \eta(x, y))\},
$$

i.e.,

$$
f(y+\lambda \eta(x, y)) \leq f\left(\gamma+\lambda^{*} \eta(x, y)\right), \quad \forall \lambda \in[0,1] .
$$


Hence,

$$
f_{+}^{\prime}\left(y+\lambda^{*} \eta(x, y) ; \eta(x, y)\right)=\limsup _{t \rightarrow 0^{+}} \frac{f\left(y+\left(\lambda^{*}+t\right) \eta(x, y)\right)-f\left(y+\lambda^{*} \eta(x, y)\right)}{t} \leq 0 \text { (3.3) }
$$

Since $\eta$ satisfies Condition $C$ and $f_{+}^{\prime}$ is subodd in the second argument, then (3.3) implies

$$
f_{+}^{\prime}\left(y+\lambda^{*} \eta(x, y) ; \eta\left(y, y+\lambda^{*} \eta(x, y)\right)\right) \geq 0
$$

According to the pseudoinvexity of $f$, we have

$$
f(y) \geq f\left(y+\lambda^{*} \eta(x, y)\right)
$$

From (3.1) and (3.2), we get

$$
f(y)<f\left(y+\lambda^{*} \eta(x, y)\right)
$$

which contradicts (3.4). Hence, $f$ is a prequasiinvex function with respect to $\eta$ on $\Gamma$.

Theorem 3.2. Let $\Gamma$ be an $\eta$-invex subset of $R^{n}$, and let $\eta: \Gamma \times \Gamma \rightarrow R^{n}$ be a given mapping. Suppose that

(1) $f: \Gamma \rightarrow R$ is radially upper semicontinuous on $\Gamma$;

(2) $f$ and $\eta$ satisfy Conditions $A$ and $C$, respectively;

(3) $f_{+}^{\prime}$ is subodd in the second argument.

Then, $f$ is pseudoinvex with respect to $\eta$ on $\Gamma$ if and only if $f_{+}^{\prime}$ is $\eta$-pseudomonotone on $\Gamma$.

Proof. Suppose that $f$ is pseudoinvex with respect to $\eta$ on $\Gamma$. Let $x, y \in \Gamma$,

$$
f_{+}^{\prime}(x, \eta(y, x)) \geq 0 \text {. }
$$

In order to show $f_{+}^{\prime}$ is $\eta$-pseudomonotone on $\Gamma$, we need to show $f_{+}^{\prime}(y, \eta(x, y)) \leq 0$. Assume, on the contrary,

$$
f_{+}^{\prime}(y ; \eta(x, y))>0
$$

According to the pseudoinvexity of $f$, from (3.5), we get $f(y) \geq f(x)$, from (3.6), we get $f(x) \geq f(y)$. Hence, $f(x)=f(y)$. By Theorem 3.1, we know that $f$ is also prequasiinvex with respect to the same $\eta$ on $\Gamma$, which implies

$$
f(y+\lambda \eta(x, y)) \leq f(x)=f(y), \quad \forall \lambda \in[0,1]
$$

Consequently,

$$
f_{+}^{\prime}(y ; \eta(x, y))=\limsup _{t \rightarrow 0^{+}} \frac{f(y+t \eta(x, y))-f(y)}{t} \leq 0,
$$

which contradicts (3.6).

Conversely, suppose that $f_{+}^{\prime}$ is $\eta$-pseudomonotone on $\Gamma$. Let $x, y \in \Gamma$,

$$
f_{+}^{\prime}(y ; \eta(x, y)) \geq 0
$$

In order to show that $f$ is a pseudoinvex function with respect to $\eta$ on $\Gamma$, we need to show $f(x) \geq f(y)$. Assume, on the contrary, 


$$
f(x)<f(y) \text {. }
$$

According to the mean value Theorem 2.1, $\exists \bar{\lambda} \in[0,1), u=y+\bar{\lambda} \eta(x, y)$ such that

$$
f(x)-f(y) \geq f_{+}^{\prime}(u ; \eta(x, y)) .
$$

From (3.8) and (3.9), we get

$$
f_{+}^{\prime}(u ; \eta(x, y))<0 .
$$

Note that $u=y+\bar{\lambda} \eta(x, y)$, if $\bar{\lambda}=0$, from (3.10), we get $f_{+}^{\prime}(y ; \eta(x, y))<0$, which contradicts (3.7). Hence, $\bar{\lambda} \in(0,1)$. Since $f_{+}^{\prime}$ is subodd in the second argument, (3.10) implies

$$
f_{+}^{\prime}(u ;-\eta(x, y))>0 .
$$

It follows from $(3.11)$ and $\bar{\lambda} \in(0,1)$ that $f_{+}^{\prime}(u ;-\bar{\lambda} \eta(x, y))>0$. By Condition $C$, we get

$$
f_{+}^{\prime}(u ; \eta(y, u))>0 .
$$

From the $\eta$-pseudomonotonicity of $f_{+}^{\prime}$, we get $f_{+}^{\prime}(\gamma ; \eta(u, y))<0$. Again using the Condition C, we obtain $f_{+}^{\prime}(y ; \eta(x, y))<0$, which contradicts (3.7).

Theorem 3.3. Let $f: \Gamma \rightarrow R$ be radially upper semicontinuous on the $\eta$-invex set $\Gamma$. Suppose that

(1) $f$ is a pseudoinvex function with respect to $\eta$ on $\Gamma$;

(2) $f$ and $\eta$ satisfy Conditions $A$ and $C$, respectively;

(3) $f_{+}^{\prime}$ is subodd in the second argument.

Then, $f$ is semistrictly prequasiinvex with respect to the same $\eta$ on $\Gamma$.

Proof. Suppose, on the contrary, $f$ is not a semistrictly prequasinvex function with respect to $\eta$ on $\Gamma$, then there exist points $x, y \in \Gamma$ with $f(x) \neq f(y)$ and $\bar{\lambda} \in(0,1)$ such that

$$
f(y+\bar{\lambda} \eta(x, y)) \geq \max \{f(x), f(y)\} .
$$

Without loss of generality, let $f(y)>f(x)$, then

$$
f(y+\bar{\lambda} \eta(x, y)) \geq f(y)>f(x) .
$$

By the mean value Theorem 2.1, $\exists \lambda^{*} \in(0,1), u=\gamma+\bar{\lambda} \eta(x, y)+\lambda^{*} \eta(\gamma, y+\bar{\lambda} \eta(x, y))$ such that

$$
0 \geq f(y)-f(y+\bar{\lambda} \eta(x, y)) \geq f_{+}^{\prime}(u ; \eta(\gamma, y+\bar{\lambda} \eta(x, y))) .
$$

According to Condition $C$ and (3.13), we get $f_{+}^{\prime}(u ;-\bar{\lambda} \eta(x, y)) \leq 0$. By the suboddity and positively homogeneity of $f_{+}^{\prime}$ in the second argument, we have

$$
f_{+}^{\prime}(u ; \eta(x, y)) \geq 0 .
$$

Note that $u=y+\bar{\lambda}\left(1-\lambda^{*}\right) \eta(x, y)$, if $\lambda^{*}=0$, then $u=y+\bar{\lambda} \eta(x, y)$. From (3.13), we get

$$
0 \geq f_{+}^{\prime}(y+\bar{\lambda} \eta(x, y) ; \eta(y, y+\bar{\lambda} \eta(x, y))) .
$$


Since $f_{+}^{\prime}$ is subodd and positively homogeneous in the second argument, from Condition $\mathrm{C}$, we have

$$
f_{+}^{\prime}(y+\bar{\lambda} \eta(x, y) ; \eta(x, y)) \geq 0
$$

Consequently, $f_{+}^{\prime}(y+\bar{\lambda} \eta(x, y) ; \eta(x, y+\bar{\lambda} \eta(x, y)) \geq 0$. By the pseudoinvexity of $f$, $f(x) \geq f(y+\bar{\lambda} \eta(x, y)$, which contradicts (3.12). Therefore, $\lambda * \neq 0$ and $\lambda * \in(0,1)$. From (3.14), $u=y+\bar{\lambda}\left(1-\lambda^{*}\right) \eta(x, y)$, Remark 2.1 and $\lambda^{*} \in(0,1)$, we get

$$
f_{+}^{\prime}\left(y+\bar{\lambda}\left(1-\lambda^{*}\right) \eta(x, y) ; \eta\left(y+\bar{\lambda} \eta(x, y), y+\bar{\lambda}\left(1-\lambda^{*}\right) \eta(x, y)\right)\right) \geq 0
$$

By Theorem 3.2, we know $f_{+}^{\prime}$ is $\eta$-pseudomonotone on $\Gamma$. Therefore,

$$
f_{+}^{\prime}\left(y+\bar{\lambda} \eta(x, y) ; \eta\left(y+\bar{\lambda}\left(1-\lambda^{*}\right) \eta(x, y), y+\bar{\lambda} \eta(x, y)\right)\right) \leq 0 .
$$

From Condition $C$, the suboddity and positively homogeneity of $f_{+}^{\prime}$ in the second argument, we obtain

$$
f_{+}^{\prime}(y+\bar{\lambda} \eta(x, y) ; \eta(x, y)) \geq 0
$$

Hence, $f_{+}^{\prime}(y+\bar{\lambda} \eta(x, y) ; \eta(x, y+\bar{\lambda} \eta(x, y))) \geq 0$. From the pseudoinvexity of $f$, we get $f(x) \geq f(y+\bar{\lambda} \eta(x, y)$, which contradicts (3.12).

\section{Characterizations of the solution sets of pseudoinvex programs}

Consider the nonlinear optimization problem

$$
\begin{aligned}
& \text { (P) } \min f(x) \\
& \text { s.t. } x \in \Gamma,
\end{aligned}
$$

where $\Gamma$ is an $\eta$-invex subset of $R^{n}$ and $f: \Gamma \rightarrow R$ is a real-valued nondifferentiable pseudoinvex function on $\Gamma$. This class of optimization problems is called the class of pseudoinvex programs.

We assume that the solution set of $(\mathrm{P})$, denoted by

$$
S:=\operatorname{argmin}\{f(x) \mid x \in \Gamma\},
$$

is nonempty. Next, we state our main results.

Theorem 4.1. Let $f: \Gamma \rightarrow R$ be radially upper semicontinuous on the $\eta$-invex set $\Gamma$. Suppose that

(1) $f$ is a pseudoinvex function with respect to $\eta$ on $\Gamma$;

(2) $f$ and $\eta$ satisfy Conditions $A$ and $C$, respectively;

(3) $f_{+}^{\prime}$ is subodd in the second argument.

Then, the solution set $S$ of $(P)$ is an $\eta$-invex set.

Proof. Let $x, y \in S$, then for any $z \in \Gamma$,

$$
f(x)=f(y) \leq f(z)
$$

According to Theorem 3.1, we know that $f$ is a prequasiinvex function with respect to $\eta$ on $\Gamma$, which implies

$$
f(y+\lambda \eta(x, y)) \leq \max \{f(x), f(y)\} \leq f(z), \quad \forall \lambda \in[0,1], \forall z \in \Gamma .
$$


Hence, $y+\lambda \eta(x, y) S$, for any $\lambda \in[0,1]$.

Lemma 4.1. Let $f: \Gamma \rightarrow R$ be radially upper semicontinuous on the $\eta$-invex set $\Gamma$. Suppose that

(1) $f$ is a pseudoinvex function with respect to $\eta$ on $\Gamma$;

(2) $f$ and $\eta$ satisfy Conditions $A$ and $C$, respectively;

(3) $f_{+}^{\prime}$ is subodd in the second argument.

Then, $\forall x, y \in S$,

$$
f_{+}^{\prime}(x, \eta(y, x))=f_{+}^{\prime}(y, \eta(x, y))=0 .
$$

Proof. Since $x, y \in S$, we obtain

$$
\begin{aligned}
& f_{+}^{\prime}(x, \eta(y, x)) \geq 0 \\
& f_{+}^{\prime}(y, \eta(x, y)) \geq 0 .
\end{aligned}
$$

Based on the pseudoinvexity of $f$ and Theorem 3.2, we know $f_{+}^{\prime}$ is $\eta$-pseudomonotone. Hence, inequalities (4.1) and (4.2) imply, respectively,

$$
\begin{aligned}
& f_{+}^{\prime}(y, \eta(x, y)) \leq 0 \\
& f_{+}^{\prime}(x, \eta(y, x)) \leq 0 .
\end{aligned}
$$

Thus, (4.1) and (4.4) imply $f_{+}^{\prime}(x, \eta(y, x))=0,(4.2)$ and (4.3) imply $f_{+}^{\prime}(y, \eta(x, y))=0$

Theorem 4.2. Let $f: \Gamma \rightarrow R$ be radially upper semicontinuous on the $\eta$-invex set $\Gamma$, and let $\bar{x} \in$ Sbe a given point. Suppose that

(1) $f$ is a pseudoinvex function with respect to $\eta$ on $\Gamma$;

(2) $f$ and $\eta$ satisfy Conditions $A$ and $C$, respectively;

(3) $f_{+}^{\prime}$ is subodd in the second argument.

Then,

$$
S=\tilde{S}=\tilde{S}_{1}=S^{*}=S_{1}^{*}=\tilde{S} \cap \hat{S},
$$

where

$$
\begin{aligned}
\tilde{S} & :=\left\{x \in \Gamma \mid f^{\prime}{ }_{+}(x ; \eta(\bar{x}, x))=0\right\} \\
\tilde{S}_{1}: & =\left\{x \in \Gamma \mid f^{\prime}{ }_{+}(x ; \eta(\bar{x}, x)) \geq 0\right\}, \\
S^{*}: & =\left\{x \in \Gamma \mid f^{\prime}{ }_{+}(\bar{x} ; \eta(x, \bar{x}))=f^{\prime}{ }_{+}(x ; \eta(\bar{x}, x))\right\}, \\
S_{1}^{*}: & =\left\{x \in \Gamma \mid f^{\prime}{ }_{+}(\bar{x} ; \eta(x, \bar{x})) \leq f^{\prime}{ }_{+}(x ; \eta(\bar{x}, x))\right\}, \\
\hat{S}: & =\left\{x \in \Gamma \mid f^{\prime}{ }_{+}(\bar{x} ; \eta(x, \bar{x}))=0\right\} .
\end{aligned}
$$

Proof. (i) It is obvious that $\tilde{S} \cap \hat{S} \subseteq \tilde{S} \subseteq \tilde{S}_{1}$ and $\tilde{S} \cap \hat{S} \subseteq S^{*} \subseteq S_{1}^{*}$.

(ii) We prove that $S \subseteq \tilde{S} \cap \hat{S}$. Let $x \in S$. From $x, \bar{x} \in S$ and Lemma 4.1, we get $f_{+}^{\prime}(\bar{x} ; \eta(x, \bar{x}))=f_{+}^{\prime}(x ; \eta(\bar{x}, x))=0$. Hence, $x \in \tilde{S} \cap \hat{S}$. 
(iii) We prove that $S_{1}^{*} \subseteq \tilde{S}_{1}$. If $x \in S_{1}^{*}$, then $f_{+}^{\prime}(\bar{x} ; \eta(x, \bar{x})) \leq f_{+}^{\prime}(x ; \eta(\bar{x}, x))$. Since $\bar{x} \in S$, we get $f_{+}^{\prime}(\bar{x} ; \eta(x, \bar{x})) \geq 0$. Hence, $f_{+}^{\prime}(x ; \eta(\bar{x}, x)) \geq 0$. We obtain $x \in \tilde{S}_{1}$.

(iv) We prove that $\tilde{S}_{1} \subseteq S$. If $x \in \tilde{S}_{1}$, then $f_{+}^{\prime}(x ; \eta(\bar{x}, x)) \geq 0$. According to the pseudoinvex of $f$, we get $f(\bar{x}) \geq f(x)$. Since $\bar{x} \in S$, from the definition of $S$, we get $x \in S$.

Remark 4.1. If $f$ is differentiable, then $f_{+}^{\prime}(x ; \eta(y, x))=\nabla f(x)^{T} \eta(y, x)$. In this case, Lemma 4.1 recovers to Lemma 3.1 in [7], Theorem 4.2 recovers to Theorems 3.1 and $3.2 \mathrm{in}$ [7]. So, the results in Lemma 4.1 and Theorem 4.2 are the extension of the results in [7].

If we take $\eta(x, y)=x-y$ in Theorem 4.2, we obtain the following result.

Corollary 4.1. Let $f: \Gamma \rightarrow R$ be radially upper semicontinuous on convex set $\Gamma$, and let $\bar{x} \in S S$ be a given point. Suppose that

(1) $f$ is a pseudoconvex function on $\Gamma$;

(2) $f_{+}^{\prime}$ is subodd in the second argument.

Then,

$$
S=S^{1}=S_{1}^{1}=S^{2}=S_{1}^{2}=S^{1} \cap S^{3},
$$

where

$$
\begin{aligned}
& S^{1}:=\left\{x \in \Gamma \mid f^{\prime}{ }_{+}(x ; \bar{x}-x)=0\right\} \\
& S_{1}^{1}:=\left\{x \in \Gamma \mid f^{\prime}{ }_{+}(x ; \bar{x}-x) \geq 0\right\} \\
& S^{2}:=\left\{x \in \Gamma \mid f^{\prime}{ }_{+}(\bar{x} ; x-\bar{x})={f^{\prime}}_{+}(x ; \bar{x}-x)\right\} \\
& S_{1}^{2}:=\left\{x \in \Gamma \mid f^{\prime}{ }_{+}(\bar{x} ; x-\bar{x}) \leq f^{\prime}{ }_{+}(x ; \bar{x}-x)\right\} \\
& S^{3}:=\left\{x \in \Gamma \mid f^{\prime}{ }_{+}(\bar{x} ; x-\bar{x})=0\right\} .
\end{aligned}
$$

\section{Variational-like inequality}

Consider the following two classes of variational-like inequalities:

Stampacchia-type variational-like inequality (SVLI): find $\bar{x} \in \Gamma$ such that

$$
f_{+}^{\prime}(\bar{x} ; \eta(x, \bar{x})) \geq 0, \quad \forall x \in \Gamma .
$$

Minty-type variational-like inequality (MVLI): find $\bar{x} \in \Gamma$ such that

$$
f_{+}^{\prime}(\bar{x} ; \eta(\bar{x}, x)) \leq 0, \quad \forall x \in \Gamma .
$$

Next, we establish the relationships between (SVLI) and (P) and between (MVLI) and (P).

Theorem 5.1. Let be an $\Gamma$-invex subset of $R^{n}$.

(i) If $\bar{x} \in$ Tis a solution of (P), then $\bar{x}$ is a solution of (SVLI);

(ii) If $\bar{x} \in \Gamma$ is a solution of (SVLI), in addition, assume that $f$ is a pseudoinvex function with respect to on $\Gamma$, then $\bar{x}$ is a solution of $(P)$.

Proof. (i) Let $\bar{x} \in \Gamma$ be a solution of the (P), then for any $x \in \Gamma, f(x) \geq f(\bar{x})$. 
Since $\Gamma$ is an $\eta$-invex set, for any $\lambda \in(0,1)$, we have $\bar{x}+\lambda \eta(x, \bar{x}) \in \Gamma$. Hence,

$$
\frac{f(\bar{x}+\lambda \eta(x, \bar{x}))-f(\bar{x})}{\lambda} \geq 0, \quad \forall \lambda \in(0,1) .
$$

Taking the limit sup as $\lambda \rightarrow 0^{+}$, we obtain

$$
f_{+}^{\prime}(\bar{x} ; \eta(x, \bar{x}))=\limsup _{t \rightarrow 0^{+}} \frac{f(\bar{x}+\lambda \eta(x, \bar{x}))-f(\bar{x})}{\lambda} \geq 0, \quad \forall x \in \Gamma,
$$

which implies $\bar{x}$ is a solution of (SVLI).

(ii) Let $\bar{x} \in \Gamma$ be a solution of the (SVLI), then

$$
f_{+}^{\prime}(\bar{x} ; \eta(x, \bar{x})) \geq 0, \quad \forall x \in \Gamma .
$$

According to the pseudoinvexity of $f$, we obtain

$$
f(x) \geq f(\bar{x}), \quad \forall x \in \Gamma,
$$

which implies $\bar{x}$ is a solution of $(\mathrm{P})$.

Theorem 5.2. Let $f: \Gamma \rightarrow R$ be a pseudoinvex function with respect to $\eta$ on the $\eta$ invex set $\Gamma$, and let $\eta$ satisfy Condition $C$.

(i) If $\bar{x} \in$ Tis a solution of $(P)$, in addition, assume that $f$ is radially upper semicontinuous on $\Gamma, f$ satisfies Condition $A, f_{+}^{\prime}$ is subodd in the second argument, then $\bar{x}$ is a solution of $M V L I$ );

(ii) If $\bar{x} \in \Gamma$ is a solution of (MVLI), for any given $x, y \in \Gamma$, the mapping $\lambda \mapsto f_{+}^{\prime}(y+\lambda \eta(x, y) ; \eta(x, y))$ is continuous at $0^{+}$, then $\bar{x}$ is a solution of $(P)$.

Proof. (i) Suppose that $\bar{x} \in \Gamma$ is a solution of (P). By Theorem 5.1(i), $\bar{x}$ is a solution of (SVLI), i.e.,

$$
f_{+}^{\prime}(\bar{x} ; \eta(x, \bar{x})) \geq 0, \quad \forall x \in \Gamma .
$$

From the pseudoinvexity of $f$ and Theorem 3.2, we know $f_{+}^{\prime}$ is $\eta$-pseudomonotone on Г. Hence,

$$
f_{+}^{\prime}(\bar{x} ; \eta(\bar{x}, x)) \leq 0, \quad \forall x \in \Gamma .
$$

which implies $\bar{x}$ is a solution of (MVLI).

(ii) Suppose that $\bar{x} \in \Gamma$ is a solution of (MVLI), then

$$
f_{+}^{\prime}(y ; \eta(\bar{x}, y)) \leq 0, \quad \forall x \in \Gamma .
$$

Since $\Gamma$ is an $\eta$-invex set, for any $\lambda \in(0,1)$, we have $\bar{x}+\lambda \eta(y, \bar{x}) \in \Gamma$. Hence,

$$
f_{+}^{\prime}(\bar{x}+\lambda \eta(y, \bar{x}) ; \eta(\bar{x}, \bar{x}+\lambda \eta(y, \bar{x}))) \leq 0, \quad \forall y \in \Gamma, \quad \forall \lambda \in(0,1) .
$$

It follows form Condition $C$ and (5.1) that $f_{+}^{\prime}(\bar{x}+\lambda \eta(y, \bar{x}) ;-\lambda \eta(y, \bar{x})) \leq 0$. According to the suboddity and the positively homogeneity of $f_{+}^{\prime}$ in the second argument, we get

$$
\left.f_{+}^{\prime}(\bar{x}+\lambda \eta(y, \bar{x}) ; \eta(y, \bar{x}))\right) \geq 0
$$

Letting $\lambda \rightarrow 0^{+}$, by the continuity of the mapping $\lambda \mapsto f_{+}^{\prime}(y+\lambda \eta(x, y) ; \eta(x, y))$, at $0^{+}$ 
we obtain

$$
f_{+}^{\prime}(\bar{x} ; \eta(y, \bar{x})) \geq 0, \quad \forall y \in \Gamma,
$$

which implies that $\bar{x}$ is a solution of (SVLI). From the pseudoinvexity of $f$ and Theorem 5.1(ii), we obtain that $\bar{x}$ is a solution of (P).

From Theorems 4.2 and 5.1, we obtain the following theorem. Denote the solution set of (SVLI) by $S_{\text {SVLI }}$.

Theorem 5.3. Let $f: \Gamma \rightarrow R$ be radially upper semicontinuous on the $\eta$-invex set $\Gamma$, and let $\bar{x} \in S_{\mathrm{SVLI}}$ be a given point. Suppose that

(1) $f$ is a pseudoinvex function with respect to $\eta$ on $\Gamma$;

(2) $f$ and $\eta$ satisfy Conditions $A$ and $C$, respectively;

(3) $f_{+}^{\prime}$ is subodd in the second argument.

Then,

$$
S_{\mathrm{SVLI}}=\tilde{S}=\tilde{S}_{1}=S^{*}=S_{1}^{*}=\tilde{S} \cap \hat{S} .
$$

From Theorems 4.2 and 5.2, we obtain the following theorem. Denote the solution set of (MVLI) by $S_{\mathrm{MVLI}}$.

Theorem 5.4. Let $f: \Gamma \rightarrow R$ be radially upper semicontinuous on the $\eta$-invex set $\Gamma$, and let $\bar{x} \in S_{\mathrm{MVLI}}$ be a given point. Suppose that

(1) $f$ is a pseudoinvex function with respect to $\eta$ on $\Gamma$;

(2) $f$ and $\eta$ satisfy Conditions $A$ and $C$, respectively;

(3) $f_{+}^{\prime}$ is subodd in the second argument;

(4) for any given $x, y \in \Gamma$, the mapping $\lambda \mapsto f_{+}^{\prime}(y+\lambda \eta(x, y) ; \eta(x, y))$ is continuous at 0 $+$

Then,

$$
S_{\mathrm{MVLI}}=\tilde{S}=\tilde{S}_{1}=S^{*}=S_{1}^{*}=\tilde{S} \cap \hat{S} .
$$

\section{Acknowledgements}

The authors thank the referees for helpful comments. This work is supported by the National Natural Science Foundation of China (10831009), the Natural Science Foundation of China (CSTC,2011BA0030), the Research Foundation Grant of The Hong Kong Polytechnic University and the Education Committee project Research Foundation of Chongqing (China) (KJ110624).

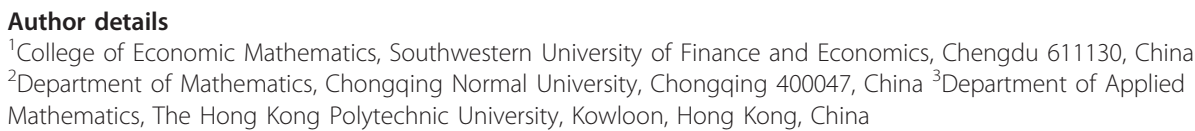

Authors' contributions

$\mathrm{CL}$ completed the main part of this article, XY presented the ideas of this article, $\mathrm{HL}$ participated in some study of this article. All authors read and approved the final manuscript.

Competing interests

The authors declare that they have no competing interests.

Received: 7 February 2011 Accepted: 17 August 2011 Published: 17 August 2011 


\section{References}

1. Mangasarian, OL: A simple characterization of solution sets of convex programs. Oper Res Lett. 7, 21-26 (1988). doi:10.1016/0167-6377(88)90047-8

2. Burke, JV, Ferris, MC: Characterization of solution sets of convex programs. Oper Res Lett. 10, $57-60$ (1991). doi:10.1016/ 0167-6377(91)90087-6

3. Jeyakumar, V: Infinite -dimensional convex programming with applications to constrained approximation. J Optim Theory Appl. 75, 469-586 (1992)

4. Jeyakumar, V, Yang, XQ: Convex composite multi-objective nonlinear programming. Math Program. 59, 325-343 (1993). doi:10.1007/BF01581251

5. Jeyakumar, V, Yang, XQ: On characterizing the solution sets of pseudolinear programs. J Optim Theory Appl. 87, 747-755 (1995). doi:10.1007/BF02192142

6. Ansari, QH, Schaible, S, Yao, JC: $\eta$-pseudolinearity. Riv Mat Sci Econ Soc. 22, $31-39$ (1999)

7. Yang, XM: On characterizing the solution sets of pseudoinvex extremum problems. J Optim Theory Appl. 140, 537-542 (2009). doi:10.1007/s10957-008-9470-7

8. Mancino, OG, Stampacchia, G: Convex programming and variational inequalities. J Optim Theory Appl. 9, 3-23 (1972). doi:10.1007/BF00932801

9. Parida, J, Sahoo, M, Kumar, A: A variational-like inequality problem. B Aust Math Soc. 39, 225-231 (1989). doi:10.1017/ 50004972700002690

10. Crespi, GP, Ginchev, I, Rocca, M: Minty variational inequalities, increase along rays property and optimization. J Optim Theory Appl. 123, 479-496 (2004). doi:10.1007/s10957-004-5719-y

11. Crespi, GP, Ginchev, I, Rocca, M: Existence of solutions and star-shapedness in minty variational inequalities. J Glob Optim. 32, 485-494 (2005). doi:10.1007/s10898-003-2685-0

12. Ivanov, VI: Optimization and variational inequalities with pseudoconvex functions. J Optim Theory Appl. 146, 602-616 (2010). doi:10.1007/s10957-010-9682-5

13. Weir, T, Mond, B: Preinvex functions in multiple objective optimization. J Math Anal Appl. 136, 29-38 (1988). doi:10.1016/0022-247X(88)90113-8

14. Mohan, SR, Neogy, SK.: On invex sets and preinvex functions. J Math Anal Appl. 189, $901-908$ (1995). doi:10.1006/ jmaa.1995.1057

15. Yang, XM, Yang, XQ, Teo, KL: Characterizations and applications of prequasi-Invex functions. J Optim Theory Appl. 110, 645-668 (2001). doi:10.1023/A:1017544513305

16. Yang, $\mathrm{XM}$, Yang, $\mathrm{XQ}, \mathrm{TeO}, \mathrm{KL}$ : Generalized invexity and generalized invariant monotonicity. J Optim Theory Appl. 117, 607-625 (2003). doi:10.1023/A:1023953823177

17. Sach, PH, Penot, JP: Charaterizations of generalized convexities via generalized directional derivative. Numer Funct Anal Optim. 19, 615-634 (1998). doi:10.1080/01630569808816849

18. Diewert, WE: Alternative charaterizations of six kinds of quasiconcavity in the nondifferentiable case with applications to nonsmooth programming. In: Schaible S, Ziemba WT (eds.) Generalized Concavity in Optimization and Economics. pp. 51-93. Academic Press, New York (1981)

19. Komlosi, S: Generalized monotonicity and generalized convexity. J Optim Theory Appl. 84, 361-376 (1995). doi:10.1007/ BF02192119

doi:10.1186/1029-242X-2011-32

Cite this article as: Liu et al:: Characterizations of the solution sets of pseudoinvex programs and variational inequalities. Journal of Inequalities and Applications 2011 2011:32.

\section{Submit your manuscript to a SpringerOpen ${ }^{\circ}$ journal and benefit from:}

- Convenient online submission

- Rigorous peer review

- Immediate publication on acceptance

- Open access: articles freely available online

- High visibility within the field

- Retaining the copyright to your article

Submit your next manuscript at $\boldsymbol{\wedge}$ springeropen.com 